\title{
ס-Opioid Receptor Activation and MicroRNA Expression in the Rat Heart Under Prolonged Hypoxia
}

\author{
Feng Zhi ${ }^{a} \quad$ Lian Xue Naiyuan Shao $^{\mathrm{a}}$ Danni Deng ${ }^{\mathrm{a}}$ Xuezhi Kang ${ }^{\mathrm{b}, \mathrm{c}}$ \\ Dongman Chao ${ }^{d}$ Yuan Xua Rong Wang ${ }^{a}$ Yilin Yang ${ }^{a}$ Ying Xia ${ }^{d}$
}

aModern Medical Research Center, The Third Affiliated Hospital of Soochow University, Changzhou, Jiangsu, 'bhanghai Research Center for Acupuncture and Meridians, Shanghai, 'Shanghai Key laboratory of Acupuncture Mechanism and Acupoint Function, Fudan University, Shanghai, China;

dDepartment of Neurosurgery, The University of Texas McGovern Medical School, Houston, Texas, USA

\section{Key Words}

$\delta$-opioid receptor $\cdot$ MicroRNA $\bullet$ Cardioprotection $\bullet$ Heart $\bullet$ Hypoxia

\begin{abstract}
Background: Hypoxic/ischemic injury to the heart is a frequently encountered clinical problem with limited therapeutic options. Since microRNAs (miRNAs) are involved in hypoxic/ischemic events, and $\delta$-opioid receptor (DOR) activation is known to protect against hypoxic/ischemic injury, we speculated on the involvement of DOR activation in altering miRNA expression in the heart under hypoxic conditions. The present study aimed to test our hypothesis. Methods: Male Sprague Dawley rats were exposed to hypoxia $\left(9.5-10 \% \mathrm{O}_{2}\right)$ for 1,5 , or 10 days with or without DOR activation. The target miRNAs were selected from TaqMan low-density array (TLDA) data and were further analyzed by quantitative real-time PCR. Results: We found that: 1) hypoxia alters the miRNA expression profiles depending on the hypoxic duration; 2) DOR activation shifts miRNA expression profiles in normoxic conditions and upregulates miR128a-3p, miR-134-5p, miR-135a, miR-193a-3p, miR-196a, miR-324-3p, and miR-338; and 3) DOR activation modifies hypoxia-induced changes in miRNA expression and increases the levels of miR-128a-3p, miR-134-5p, miR-135a, miR-193a-3p, miR-196a, miR-324-3p, miR141, miR-200b, and miR-324-3p. For example, miR-196c-5p decreased by $50 \%$ while miR$135 a-5 p$ increased 2.9 fold after 10 days under hypoxic conditions. Moreover, DOR activation further strengthened the hypoxia-induced increase of the levels of miR-7a-5p. When DOR was activated using UFP-512, the level of miR-107-3p significantly increased 1 day after the administration of UFP-512, but gradually decreased back to normal under normoxia. Conclusion: Hypoxia significantly modifies the miRNA profile in the heart, which can be mimicked or modified by DOR activation. Defining the targeted pathways that regulate the diverse cellular and molecular functions of miRNAs may provide new insights into potential therapies for hypoxic/ischemic injury of the heart.

F. Zhi and L. Xue contributed equally.

Yilin Yang, M.D.,
and Ying Xia, M.D., Ph.D.,

Modern Medical Research Center, Third Affiliated Hospital of Soochow University Changzhou 213003, Jiangsu Province (China); and Department of Neurosurgery, The University of Texas McGovern Medical School, Houston, TX 77030 (USA) E-Mail yilinyang.czfph@gmail.com / Y55738088@gmail.com / Ying.Xia@uth.tmc.edu
\end{abstract}

KARGER 


\section{Introduction}

Hypoxia is a state of low oxygen supply to the body, an organ, or a cell, which can be caused by either a decrease in the partial pressure of oxygen, inadequate oxygen transport, or the inability of tissue to take up oxygen from the blood. Hypoxia-induced myocardial injury is closely associated with cardiac disorders, and is particularly associated with myocardial infarction, myocardial ischemia, and heart failure [1-3]. However, until now, there is still no promising therapy available for the prevention and treatment of hypoxic heart injury.

MicroRNA (miRNA), a class of naturally occurring, 17-25 nucleotide noncoding small RNA, regulates the expression of genes by binding to the 3 '-untranslated regions (3'-UTR) of target mRNAs. In addition, there is growing evidence that suggests that miRNAs participate in the regulation of a range of biological processes [4, 5]. Given the multifactorial nature of cardiovascular disease, it is not surprising that miRNAs have been shown to orchestrate many aspects of heart disease development and have become important targets for therapeutic intervention [6, 7]. For example, miR-17 promotes cardiomyocyte apoptosis via targeting Stat3 in regulating ischemia/reperfusion-induced cardiac injury [8]. MicroRNA-21 has a protective effect against myocardial infarction: it reduces cardiac cell apoptosis by binding to its target gene, PDCD4 [9]. In addition, the combination of miR-21 and miR-146a can attenuate cardiac ischemic injuries during acute myocardial infarction in mice [10]. However, there is also data that shows that miR-21 plays a pro-inflammatory role in atherosclerosis patients with coronary heart disease through a TGF- $\beta 1 /$ Smad-independent pathway [11]. In addition, miR-24 inhibits cardiomyocyte apoptosis, attenuates infarct size, and reduces cardiac dysfunction by directly suppressing the pro-apoptotic protein Bim [12]. MicroRNA-132 is involved in long-term activation of $\beta$-adrenergic signaling in cardiomyocytes [13], while miR101a inhibits cardiac fibrosis by targeting TGF $\beta R I$ under hypoxia on cardiac fibroblasts [14]. In contrast, miR-133a mediates hypoxia-induced apoptosis by inhibiting TAGLN2 expression in cardiac myocytes [15]. Moreover, miR-210 can improve angiogenesis, inhibit apoptosis, and improve cardiac function during myocardial infarction [16]. The association between miRNAs and cardiomyopathy has given rise to its potential for use in clinical diagnosis, and as a therapeutic target.

Recently, we have demonstrated that the $\delta$-opioid receptor (DOR) is protective against anoxic/ischemic injury in the brain, and that its underlying mechanisms involve the regulation of ionic homeostasis and antioxidative capacity [17-21]. There are also other studies showing that DOR agonists can prolong survival of peripheral organs, such as the lungs, heart, liver, and kidney, and that DOR activation is protective against hypoxic/ischemic injury in the heart $[22,23]$. Since there is a growing body of evidence indicating that miRNAs play several regulatory roles in opioid pharmacology [24, 25], and our previous studies demonstrate the involvement of DOR in the regulation of miRNA expression in the brain and kidney [26, 27], we wanted to know whether DOR affects miRNA expression in the heart when exposed to prolonged hypoxia.

\section{Materials and Methods}

\section{Animals}

All animal procedures were performed in accordance with the NIH guidelines and were approved by the Animal Care and Use Committee of Shanghai Research Center for Acupuncture and Meridians and the Animal Experimental Committee of the Soochow University. Sprague Dawley male rats at 21 days of age were purchased from the Shanghai Experimental Animal Center of Chinese Academy of Sciences (China). Immediately after their arrival, all rats were randomly divided into 4 groups: (A) control, (B) DOR agonist (UFP-512), (C) chronic anoxia, and (D) chronic anoxia+DOR agonist (UFP-512). Each group had at least 6 animals for further experiments.

\section{KARGER}




\section{Cellular Physiology Cell Physiol Biochem 2016;39:1118-1128

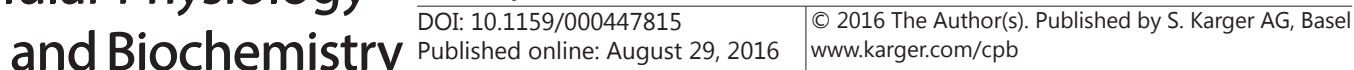 \\ Zhi et al.: $\delta$-Opioid Receptor and MicroRNAs in Hypoxic Heart}

Hypoxia induction and DOR activation

Groups A and B were raised in normal conditions, while Groups C and D were raised under hypoxic conditions. Chronic hypoxia was induced as described in our previous work [28]; the $\mathrm{O}_{2}$ level was maintained at $9.5 \%-10 \%$ in the plexiglass box to induce hypoxia. The rats of Groups C and D were kept in the hypoxic box for 1,5 , or 10 days. The box was rapidly cleaned daily when the animals were removed to have their body weights recorded. The rats of Groups B and D were subjected to an intraperitoneal injection of UFP512 (H-Dmt-Tic-NH-CH[CH2-COOH]-Bid), a specific and potent DOR agonist synthesized by our team [29]. The injections ( $1 \mathrm{mg} / \mathrm{kg}$ in $<1 \mathrm{ml}$ ) were performed on day 0 (immediately before the onset of hypoxia), day 4, and day 8. As a control, Groups A and C received the same amount of saline.

\section{Tissue Collection}

After 1, 5, or 10 days of hypoxia, the rats were decapitated following deep anesthesia. Their hearts were rapidly removed, weighed, frozen in liquid nitrogen, and stored at $-80^{\circ} \mathrm{C}$ until later use.

\section{RNA Extraction and TLDA Experiments}

The TaqMan low-density array (TLDA) assay was performed to identify the miRNAs in the heart after 10 days of hypoxia. The tissue samples from 6 rats in Group A and 6 rats in Group C at 10 days were pooled into their respective groups. Total RNA was extracted using Trizol Reagent (Invitrogen, USA) according to the manufacturer's protocol. The Taqman low density array (TLDA) experiment was performed and analyzed by CapitalBio (CapitalBio, China) as previously reported [27].

\section{Quantification of miRNAs by qRT-PCR analysis}

Whole heart homogenization was carried out under liquid nitrogen to completely disrupt all of the cells. The total RNA was extracted from the collected homogenate using TRIzol Reagent (Thermo Fisher, USA) according to the manufacturer's instructions. The miRNA expression levels were quantified using TaqMan microRNA assay (Thermo Fisher, USA) on the Applied Biosystems 7500 (Thermo Fisher, USA) as previously reported [27]. U6 served as an internal control. In brief, $1 \mu \mathrm{g}$ of total RNA was reverse transcribed to cDNA using an AMV reverse transcriptase (TaKaRa, Dalian, China) and a stem-loop primer (Applied Biosystems). The mixture was incubated at $16^{\circ} \mathrm{C}$ for $15 \mathrm{~min}, 42^{\circ} \mathrm{C}$ for $60 \mathrm{~min}$, and $85^{\circ} \mathrm{C}$ for $5 \mathrm{~min}$ to generate a library of miRNA cDNAs. qRT-PCR was then performed at $95^{\circ} \mathrm{C}$ for $10 \mathrm{~min}$, followed by 40 cycles at $95^{\circ} \mathrm{C}$ for $15 \mathrm{~s}$ and at $60^{\circ} \mathrm{C}$ for $60 \mathrm{~s}$. After the reactions were completed, the threshold cycle $(\mathrm{Ct})$ values were determined using the default threshold settings for analyzing the data. The relative amount of one miRNA to internal control U6 transcript was calculated using the equation $2^{-\Delta \mathrm{CT}}$ in which $\Delta \mathrm{C}_{\mathrm{T}}=\mathrm{C}_{\mathrm{T} \text { miRNA }}-\mathrm{C}_{\mathrm{T} \text { U6 }}$. All reactions, including the controls that contained no template RNA, were performed in triplicate.

Statistical analysis

Results are presented as the mean \pm SD with a minimum $n=6$. The mean value for Group A (the control) for each time point is set at one. Statistical significance was determined using either a student's t-test or a one-way ANOVA following Turkey's Multiple Comparison Test on paired columns as appropriate.

\section{Results}

\section{Hypoxia-induced changes in body and heart weights}

Changes in body weight and heart weight were monitored in all animals. Heart weight increased with prolonged exposure; and exposure to a hypoxic environment did not reduce weight gain by the heart in both UFP-512 treated and control animals at 24 hours after the onset of hypoxia. Small but significant differences in heart weight were noted at 5-days, between normoxic and hypoxic animals treated with UFP-512 (Fig. 1A). The heart/ body weight ratio decreased after 10 days of hypoxia, but not after exposure for 1-5 day(s), suggesting that adaptive mechanisms were acting to protect the heart from atrophy during prolonged hypoxia. Although UFP-512 did not have a detectable effect on the absolute heart weight, the addition of UFP-512 preserved more of the heart/body weight ratio under hypoxic conditions when compared to hypoxia exposure, especially after exposure for 10 days (Fig. 1B). 
Fig. 1. Effect of hypoxia and DOR activation on the heart weight. (A) Heart weight (n = 9). (B) Ratio of heart to body weight for each study group ( $\mathrm{n}=9$ ). C: control; C+DOR: control+DOR agonist UFP-512; H: hypoxia; H+DOR: hypoxia+ DOR agonist UFP-512. ${ }^{*} \mathrm{p}<0.05$.
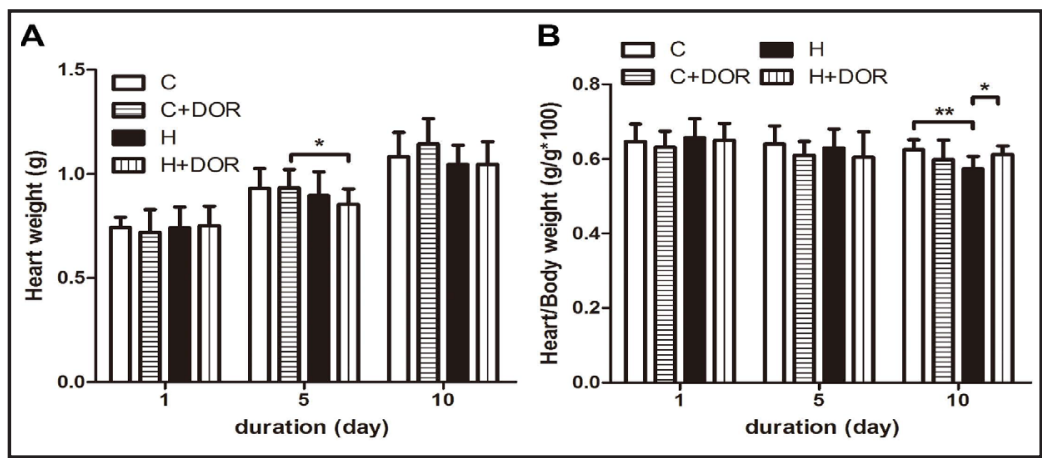

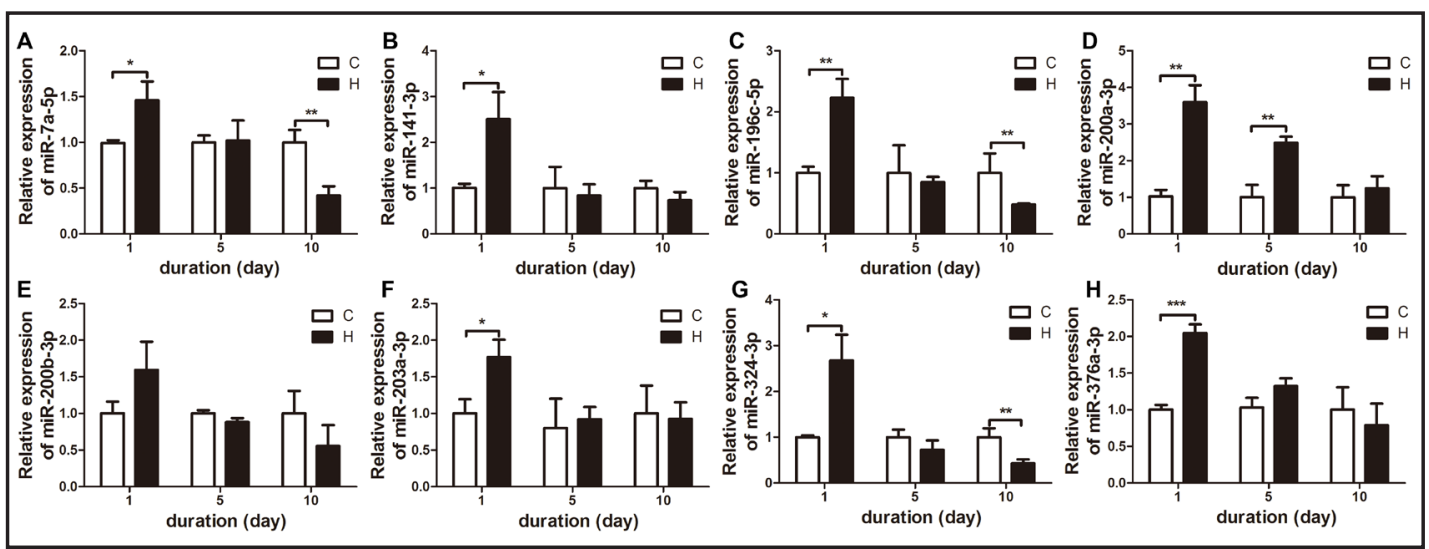

Fig. 2. Differential changes in various miRNAs in hypoxia. The expression levels of miR-7a-5p, miR-141-3p, miR-196c-5p, miR-200a-3p, miR-200b-3p, miR-203a-3p, miR-324-3p, and miR-376a-3p in the heart following 1,5 , or 10 days of hypoxia are determined by qRT-PCR. C: control; H: hypoxia. ${ }^{*} \mathrm{p}<0.05,{ }^{* *} \mathrm{p}<0.01$, $* * * \mathrm{p}<0.001$.

\section{MicroRNA TLDA analysis}

To identify the miRNA changes induced by hypoxia, comparative miRNA TLDA analysis was performed on RNA isolated from the hearts of rats exposed to either 10-day hypoxia or normoxia. The results revealed that the miRNA expression profiles varied between the hypoxic and the normoxic groups. We next narrowed down the list of miRNAs to be used in further experiments. The following criteria were used to select the miRNA for further analysis: 1) the miRNAs that are exclusively expressed in rats, 2) a fold change in the TLDA was $>200$, and 3) the assays were linear. Consequently, 17 miRNAs that met the inclusion criteria were chosen for further qRT-PCR analysis in all the samples, and include miR-7a-5p, miR-7b, miR-107-3p, miR-128-3p, miR-134-5p, miR-135a-5p, miR-141-3p, miR-193a-3p, miR-196c-5p, miR-200a-3p, miR-200b-3p, miR-203a-3p, miR-324-3p, miR-324-5p, miR338-3p, miR-350, and miR-376a-3p.

\section{Early longitudinal miRNA changes in response to hypoxia}

Hypoxia alone significantly increased the expression of miR-7a-5p, miR-141-3p, miR196c-5p, miR-200a-3p, miR-203a-3p, miR-324-3p, and miR-376a-3p in the heart after exposure for one day (Fig. 2). Hypoxia also tended to increase the level of miR-200b-3p after one day, but the change was not statistically significant (Fig. 2E). This increase in the above miRNAs was attenuated after 5 days of exposure to hypoxia. At day 10, the levels of some miRNAs (miR-7a-5p, miR-196c-5p, and miR-324-3p) were statistically lower in hypoxic animals than in normoxic animals (Fig. 2A, 2C, 2G). However, some other miRNAs (e.g., miR141-3p, miR-200b-3p, miR-203a-3p, and miR-376a-3p) were also lower in hypoxic animals, but the differences were not statistically different when compared to the control (Fig. 2B, 2E, $2 \mathrm{~F}, 2 \mathrm{H}$ ). Interestingly enough, hypoxia increased the expression of miR-200a-3p by 3.6 fold 


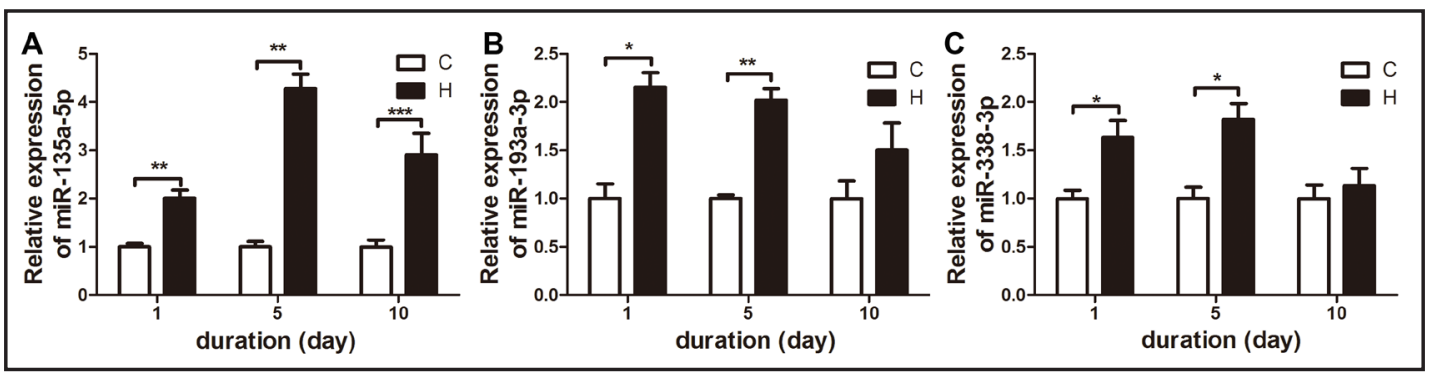

Fig. 3. Similar changes in some miRNAs in response to hypoxia. The expression levels of miR-135a-5p, miR193a-3p and miR-338-3p in the heart following 1, 5, or 10 days of hypoxia are determined by qRT-PCR. C: control; H: hypoxia. ${ }^{*} \mathrm{p}<0.05,{ }^{* *} \mathrm{p}<0.01,{ }^{* * *} \mathrm{p}<0.001$.
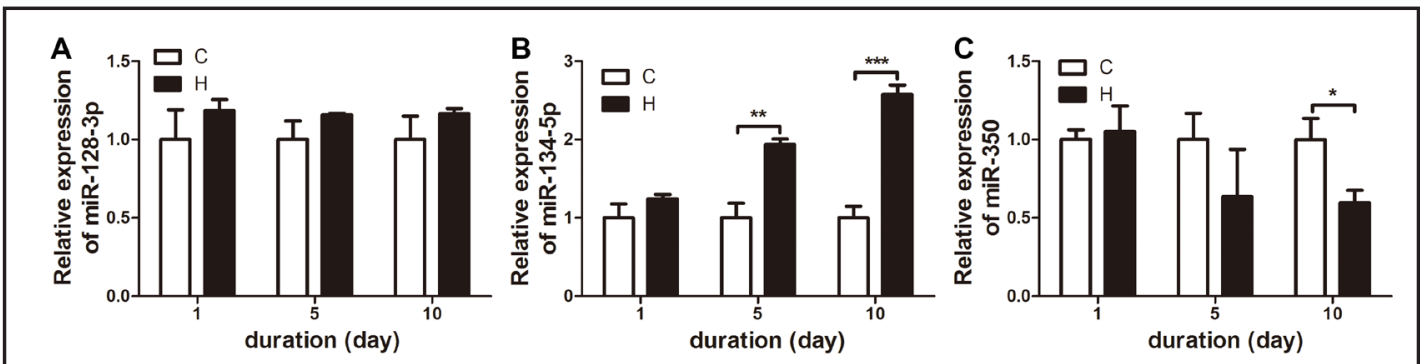

Fig. 4. Late longitudinal miRNA changes in response to hypoxia. The miRNA expression of miR-128-3p, miR-134-5p, and miR-350 was detected in the heart following 1, 5, or 10 days of hypoxia. C: control; H: hypoxia. ${ }^{*} \mathrm{p}<0.05,{ }^{* *} \mathrm{p}<0.01,{ }^{* * *} \mathrm{p}<0.001$.

after one day and 2.5 fold after 5 days. Conversely, unlike the other miRNAs described above, miR-200a-3p expression level was still higher than the basal level after exposure to hypoxia for 10 days (Fig. 2D).

On the other hand, some miRNAs share a different expression pattern. For example, hypoxia increased the level of miR-135a-5p at the first time point (1 day) and this change reached a peak after 5 days of exposure to hypoxia. The level then began to decrease after 10 days of exposure to hypoxia (Fig. 3A). miR-193a-3p and miR-338-3p reached their peaks at the first time point and this change persisted after 5 days of exposure to hypoxia. The levels then began to decrease after 10 days of exposure to hypoxia (Fig. 3B-3C).

\section{Late longitudinal miRNA changes in response to hypoxia}

The levels of expression of miR-134-5p and miR-350 showed no appreciable change following exposure to hypoxia for 1 day, whereas a longer duration ( 5 or 10 days) of treatment demonstrated marked alterations in their expression levels in the heart. miR134-5p gradually increased to about 2.7 fold of the control level under prolonged hypoxia for 10 days, while miR-350 gradually decreased to about only $60 \%$ of the control level after 10 days of hypoxia (Fig. 4A-4B). It is also interesting to note that miR-128-3p remained at a steady level in the heart under prolonged hypoxia with a slight increase at day 1 of hypoxia (Fig. 4C).

\section{MicroRNA changes in response to DOR activation under normoxia}

Some miRNAs' levels were significantly altered once DOR was activated by the administration of UFP-512. For example, the activation of DOR significantly increased the expression of miR-107-3p, miR-141-3p, and miR-350 in the heart one day after treatment using UFP-512 (one time treatment). This increase was attenuated after 5 days (2-time treatment) and completely disappeared after 10 days (3-time treatment). In fact, the levels of these three miRNAs were statistically lower in the animals treated with UFP-512 for 
Fig. 5. Effects of DOR activation on the expression of miR-10 7-3p, miR-128a-3p, miR-141-3p, and miR-350 in the heart. C: control; $\mathrm{C}+\mathrm{DOR}$ : control+DOR agonist UFP512. $* \mathrm{p}<0.05$, $* * \mathrm{p}<0.01$, $* * * \mathrm{p}<0.001$.
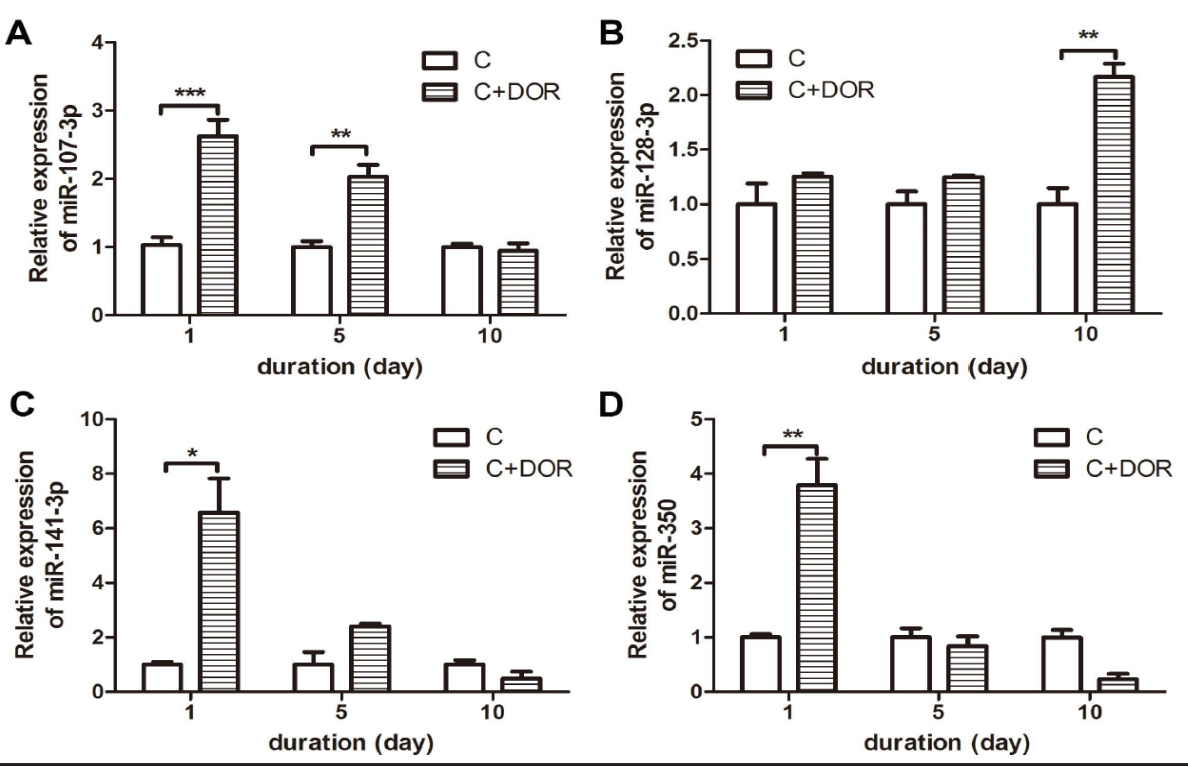

Fig. 6. Effects of DOR activation on the expression of miR - $7 \mathrm{a}-5 \mathrm{p}$, miR-107-3p, miR-200b-3p, and miR-376a$3 p$ in the hypoxic heart. C: control; H: hypoxia; H+DOR: hypoxia+ DOR agonist UFP512. $* \mathrm{p}<0.05$, $* * \mathrm{p}<0.01$, $* * * \mathrm{p}<0.001$.
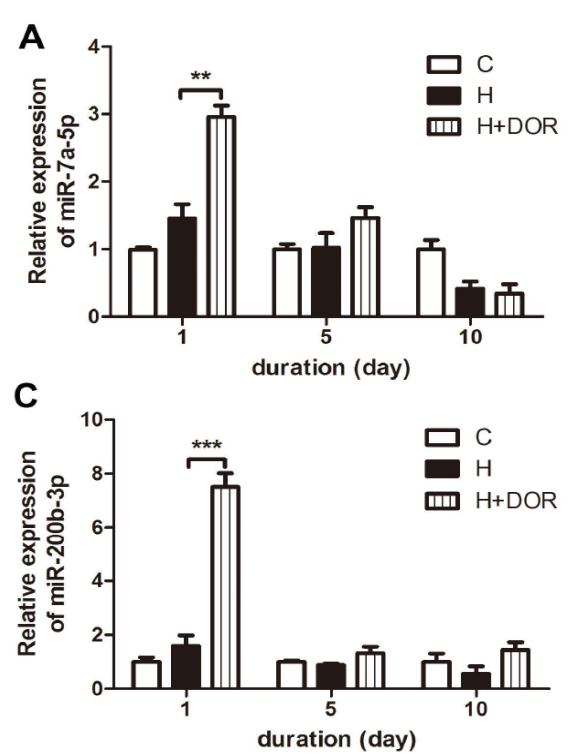
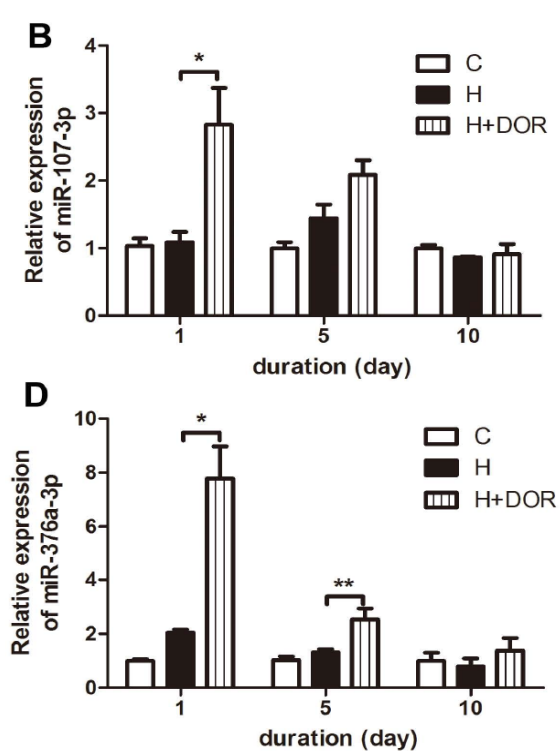

3 times (10 days) than those in normoxic animals (Fig. 5A-5C). miR-128-3p was the only miRNA whose expression level was unaltered during the first 5 days after administration, while its level was increased at day 10 of administration (Fig. 5D). All other miRNAs studied in this work remained unaltered or showed no statistical significance in response to DOR activation in the heart.

\section{MicroRNA changes in response to DOR activation under hypoxia}

Next we investigated whether DOR activation could modify hypoxia-induced changes in miRNA expression. Some miRNAs were significantly altered at the earliest time point (1 day) when UFP-512 treatment was combined with hypoxia. The combination of hypoxia and DOR activation significantly increased the expression of miR-7a-5p, miR-107-3p, miR-200b-3p, and miR-376a-3p in the heart after treatment using UFP-512 for one day. The relative levels of these miRNAs were then attenuated after 5 days of hypoxia and reached their lowest point at day 10 in response to DOR activation under hypoxia (Fig. 6). At day 10, the relative level of miR-7a-5p was lower than in the group of hypoxia alone (Fig. 6A), while miR-107-3p, 
Fig. 7. Effects of DOR activation on the expression of miR-7b and miR134-5p in the hypoxic heart. C: control; H: hypoxia; H+DOR: hypoxia+ DOR agonist UFP512. ${ }^{* *} \mathrm{p}<0.01$, $* * * \mathrm{p}<0.001$.

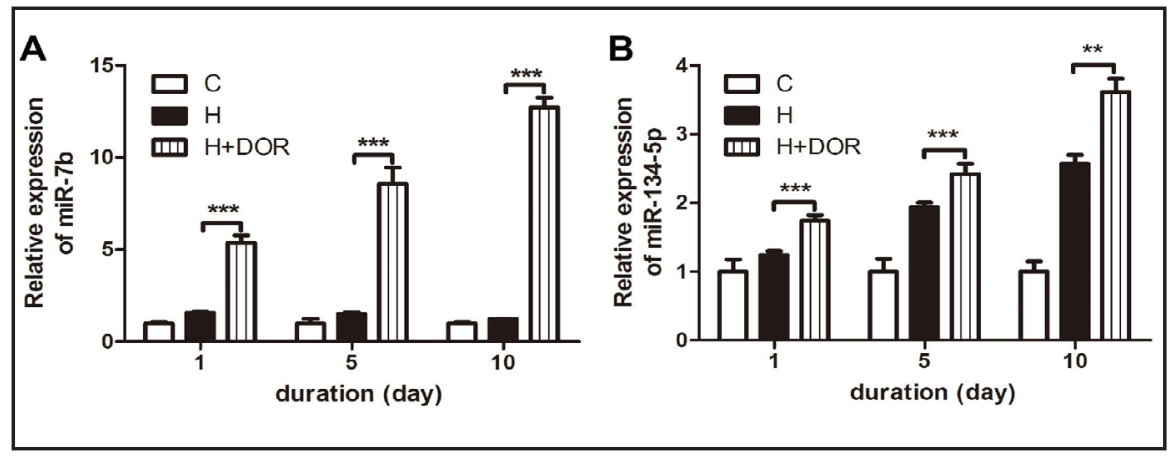

miR-200b-3p, and miR-376a-3p levels were still higher than in the group of hypoxia alone (Fig. 6B-6D). miR-7b and miR-134-5p were the only two miRNAs whose expression levels were increased throughout the entire time course (Fig. 7). At day 10, miR-7b increased about 12 fold, while miR-134-5p increased about 3.6 fold compared to their respective normoxic controls.

\section{Discussion}

Myocardial hypoxia triggers cell injury and apoptosis, which is involved in the pathogenesis of many cardiovascular diseases including myocardial ischemia, reperfusion injury, and heart failure [30]. Growing evidence indicates that miRNAs are critical regulators of cardiovascular development and physiology, and are thus directly involved in the pathophysiology of many cardiovascular diseases [31]. MicroRNAs are reported to be involved in cardiac reactions to myocardial infarction and heart failure [32]. They are powerful modifiers of cardiac aging, which is one of the most hazardous risks for cardiovascular diseases [33]. In this study, we present the first data to identify several cardiac miRNAs showing a major alteration in their expression in response to chronic hypoxia and DOR activation. Our comprehensive data demonstrated that in the rat heart: 1) hypoxia has a major effect on the expression of 17 miRNAs with a major difference in the direction and amplitude of the changes after hypoxic exposure for different durations; and 2) several miRNAs respond remarkably to DOR activation under normoxia and/or hypoxia. These results suggest that some miRNAs are very sensitive to oxygen deprivation and they may play a role in DOR-mediated cardioprotection in both normoxic and hypoxic conditions.

Over the past decade, many miRNAs and their target genes have been identified in various cardiovascular diseases by using various cellular, animal disease or transgenic models [34]; these diseases include hypertrophy, cardiac fibrosis, and cardiomyocyte apoptosis. MicroRNAs can often coordinate multiple genes in a single pathway through the regulation of gene expression in post-transcriptional pathways [35]. These findings suggest that miRNAs may be developed as diagnostic, prognostic, or therapeutic tools in cardiovascular diseases. Our study also reveals the potential of these small gene regulators as important factors involved in the hypoxic response and DOR activation in the heart.

Some miRNAs' levels were significantly altered after only one day of hypoxia, indicating that these miRNAs are sensitive to hypoxia and involved in hypoxic regulation of cellular and molecular processes in the heart. For example, miR-7a-5p is sensitive to ischemia/ reperfusion (I/R) insult and plays a role in protecting myocardial cells against I/R-induced apoptosis by negatively regulating PARP expression [36]. miR-107-3p, which is up-regulated in hypoxia as shown in this study, also serves as a protective regulator by preventing endothelial progenitor cells from differentiation by targeting HIF-1 $\beta$ [37]. Indeed, the endothelial progenitor cell is an important regulator in tissue repair after ischemic heart disease, depending on its ability to differentiate into mature endothelial cells. Unlike our findings, which show the upregulation of miR-107-3p after 1-5 day(s) of hypoxia, a previous 
study showed its downregulation in peripheral blood mononuclear cells in ischemic and dilated cardiomyopathy patients, which has potential in diagnostic and/or prognostic use in chronic heart failure [38].

The difference between our study and Voellenkle et al.'s work [38] in terms of the change in miR-107-3p expression of in response to hypoxia is likely due to the different sources of miR-107-3p: one comes from the heart tissue, and the other comes from peripheral blood. As most circulating miRNAs are actively released by cells, it is conceivable that there is a connection between tissue miR-107-3p and circulating miR-107-3p [39].

Baseler et al. observed that diabetes-induced miR-141-3p expression can influence mitochondrial proteomes and functional processes by regulating Slc25a3 protein expression in the diabetic heart [40]. Moreover, miR-200b-3p is sensitive to hypoxia and is involved in the induction of angiogenesis by directly targeting Ets-1 [41]. On the other hand, miR338-3p is correlated with off-pump cardiac index values in transplanted hearts of heart failure patients with left ventricular assist device (LVAD) assistance when compared to those without LVAD assistance [42]. These miRNAs play important roles for the heart in the initial phase of hypoxia by targeting their respective targets.

In contrast to the miRNAs described above, some miRNAs changed after exposure to hypoxia for a long time, such as miR-134-5p and miR-350. MicroRNA-134-5p is associated with an increased risk of mortality or development of heart failure and could be a potential indicator for acute myocardial infarction [43]. Circulating miR-134-5p is a novel biomarker for the early diagnosis of acute myocardial infarction [44]. It is also a potential plasma biomarker for the diagnosis of acute pulmonary embolism [45], and its increase after 5-10 days of hypoxia suggests its involvement in hypoxic heart injury. Similarly, miR-350 is a critical regulator of pathological cardiac hypertrophy in the heart and its downregulation can reduce heart cell size and apoptosis [46]. However, the detailed mechanisms behind the delayed response of these miRNAs to hypoxia in the heart still needs to be further explored.

DOR has been established as an effective regulator against hypoxia-induced injury. DOR signaling acts at multiple levels, including the inhibition of hypoxic/ischemic disruption of ionic homeostasis $[20,29,47]$, and the regulation of survival/death signals [48, 49]. Estrada et al. found that DOR protected canine myocardium from cardiac injury under intermittent hypoxia, which demonstrated the important roles of DOR in cardioprotection against hypoxia [50]. In this study, several miRNAs were identified that respond to DOR activation under normoxic and/or hypoxic conditions and most of them were increased in response to DOR activation at various time points, suggesting that DOR signaling regulates cardiac miRNAs. This may be important for cardioprotection against hypoxic/ischemic injury. For example, miR-128-3p remained at a steady level in the heart under prolonged hypoxia, but it was significantly upregulated after DOR activation. Witman et al., reported that miR128-3p was significantly elevated during newt cardiac regeneration [51], suggesting that its upregulation is of benefit to cardiac cell viability. Furthermore, its upregulation by DOR activation supported the notion that DOR is cardioprotective against hypoxic injury. We believe that such protection is at least partially related to an increase in miR-128-3p activity. However, how DOR activation regulates miRNA expression and function is still poorly known. In fact, this is the first study on the DOR-mediated regulation of miRNAs in the heart. It is necessary in future studies to use diversified techniques to acquire a better understanding of the miRNA mechanism underlying DOR-mediated protection of the heart.

In summary, we have identified a subset of cardiac miRNAs that are remarkably altered in response to hypoxia and/or DOR activation with several miRNAs increased in response to DOR activation under prolonged hypoxia. It is likely that the changes in miRNA expression alter molecular pathways influencing survival and death signaling in the heart. Further studies for in-depth understanding of the multifaceted effects of theses miRNAs on gene modulation and post-transcriptional modulation in the heart may provide an insight into new strategies for cardiac protection against hypoxic/ischemic injury. 


\section{Cellular Physiology Cell Physiol Biochem 2016;39:1118-1128 \begin{tabular}{ll|l} 
and Biochemistry & $\begin{array}{l}\text { DOI: 10.1159/000447815 } \\
\text { Published online: August 29, } 2016\end{array}$ & $\begin{array}{l}\text { C } 2016 \text { The Author(s). Published by S. Karger AG, Basel } \\
\text { www.karger.com/cpb }\end{array}$ \\
\hline
\end{tabular}

\section{Acknowledgements}

YY was supported by National Science Foundation of China (31071046), Jiangsu Provincial Special Program of Medical Science (BL2014035), Changzhou Science Development Project (CE20155060). FZ was supported by National Natural Science Foundation of China (81302197) and Changzhou High-Level Medical Talents Training Project (2016CZBJ006). XK was supported by 973 Program (2012CB518502) and NSFC (81574053; 81590953) of China. YX was supported by Memorial Hermann's Foundation and Vivian L Smith Neurologic Foundation. The funders had no role in study design, data collection and analysis, decision to publish, or preparation of the manuscript.

\section{Disclosure Statement}

The authors have declared no conflicts of interest.

\section{References}

1 Cassavaugh J, Lounsbury KM: Hypoxia-mediated biological control. J Cell Biochem 2011;112:735-744.

2 Dirksen MT, Laarman GJ, Simoons ML, Duncker DJ: Reperfusion injury in humans: A review of clinical trials on reperfusion injury inhibitory strategies. Cardiovasc Res 2007;74:343-355.

3 Frohlich GM, Meier P, White SK, Yellon DM, Hausenloy DJ: Myocardial reperfusion injury: Looking beyond primary pci. Eur Heart J 2013;34:1714-1722.

$4 \quad$ Esteller M: Non-coding rnas in human disease. Nat Rev Genet 2011;12:861-874.

5 Bartel DP: Micrornas: Genomics, biogenesis, mechanism, and function. Cell 2004;116:281-297.

6 Latronico MV, Catalucci D, Condorelli G: Emerging role of micrornas in cardiovascular biology. Circ Res 2007;101:1225-1236.

7 Small EM, Frost RJ, Olson EN: Micrornas add a new dimension to cardiovascular disease. Circulation 2010;121:1022-1032.

8 Du W, Pan Z, Chen X, Wang L, Zhang Y, Li S, Liang H, Xu C, Zhang Y, Wu Y, Shan H, Lu Y: By targeting stat3 microrna-17-5p promotes cardiomyocyte apoptosis in response to ischemia followed by reperfusion. Cell Physiol Biochem 2014;34:955-965.

9 Dong S, Cheng Y, Yang J, Li J, Liu X, Wang X, Wang D, Krall TJ, Delphin ES, Zhang C: Microrna expression signature and the role of microrna- 21 in the early phase of acute myocardial infarction. J Biol Chem 2009;284:29514-29525.

10 Huang W, Tian SS, Hang PZ, Sun C, Guo J, Du ZM: Combination of microrna-21 and microrna-146a attenuates cardiac dysfunction and apoptosis during acute myocardial infarction in mice. Molecular therapy Nucleic acids DOI: 10.3390/ijms17050749.

11 Li S, Fan Q, He S, Tang T, Liao Y, Xie J: Microrna-21 negatively regulates treg cells through a tgf-beta1/smadindependent pathway in patients with coronary heart disease. Cell Physiol Biochem 2015;37:866-878.

12 Qian L, Van Laake LW, Huang Y, Liu S, Wendland MF, Srivastava D: Mir-24 inhibits apoptosis and represses bim in mouse cardiomyocytes. J Exp Med 2011;208:549-560.

13 Carrillo ED, Sampieri R, Hernandez A, Garcia MC, Sanchez JA: Mir-132 regulates rem expression in cardiomyocytes during long-term beta-adrenoceptor agonism. Cell Physiol Biochem 2015;36:141-154.

14 Zhao X, Wang K, Liao Y, Zeng Q, Li Y, Hu F, Liu Y, Meng K, Qian C, Zhang Q, Guan H, Feng K, Zhou Y, Du Y, Chen Z: Microrna-101a inhibits cardiac fibrosis induced by hypoxia via targeting tgfbetari on cardiac fibroblasts. Cell Physiol Biochem 2015;35:213-226.

15 Li AY, Yang Q Yang K: Mir-133a mediates the hypoxia-induced apoptosis by inhibiting tagln2 expression in cardiac myocytes. Mol Cell Biochem 2015;400:173-181.

16 Hu S, Huang M, Li Z, Jia F, Ghosh Z, Lijkwan MA, Fasanaro P, Sun N, Wang X, Martelli F, Robbins RC, Wu JC: Microrna-210 as a novel therapy for treatment of ischemic heart disease. Circulation 2010;122:S124-131.

17 Zhang J, Qian H, Zhao P, Hong SS, Xia Y: Rapid hypoxia preconditioning protects cortical neurons from glutamate toxicity through delta-opioid receptor. Stroke 2006;37:1094-1099. 


\section{Cellular Physiology Cell Physiol Biochem 2016;39:1118-1128 \begin{tabular}{l|l|l|}
\hline DOI: 10.1159/000447815 29,2016 & $\begin{array}{l}\text { () 2016 The Author(s). Published by S. Karger AG, Basel } \\
\text { www.karger.com/cpb }\end{array}$
\end{tabular} \\ Zhi et al.: $\delta$-Opioid Receptor and MicroRNAs in Hypoxic Heart}

18 Zhang J, Haddad GG, Xia Y: Delta-, but not mu- and kappa-, opioid receptor activation protects neocortical neurons from glutamate-induced excitotoxic injury. Brain Res 2000;885:143-153.

19 Yang Y, Xia X, Zhang Y, Wang Q Li L, Luo G, Xia Y: Delta-opioid receptor activation attenuates oxidative injury in the ischemic rat brain. BMC Biol 2009; 7:55.

20 Chao D, Balboni G, Lazarus LH, Salvadori S, Xia Y: Na+ mechanism of delta-opioid receptor induced protection from anoxic k+ leakage in the cortex. Cell Mol Life Sci 2009;66:1105-1115.

21 Xia Y: Neural functions of the delta-opioid receptor. (New York, Heidelberg, Dordrecht, London), Springer, 2015, pp 1-728.

22 Patel HH, Head BP, Petersen HN, Niesman IR, Huang D, Gross GJ, Insel PA, Roth DM: Protection of adult rat cardiac myocytes from ischemic cell death: Role of caveolar microdomains and delta-opioid receptors. Am J Physiol Heart Circ Physiol 2006;291:H344-350.

23 Borlongan CV, Wang Y, Su TP: Delta opioid peptide (d-ala 2, d-leu 5) enkephalin: Linking hibernation and neuroprotection. Front Biosci 2004;9:3392-3398.

24 Wu Q, Hwang CK, Zheng H, Wagley Y, Lin HY, Kim do K, Law PY, Loh HH, Wei LN: Microrna 339 downregulates mu-opioid receptor at the post-transcriptional level in response to opioid treatment. FASEB J 2013;27:522-535.

25 He Y, Yang C, Kirkmire CM, Wang ZJ: Regulation of opioid tolerance by let-7 family microrna targeting the mu opioid receptor. J Neurosci 2010;30:10251-10258.

26 Yang Y, Zhi F, He X, Moore ML, Kang X, Chao D, Wang R, Kim DH, Xia Y: Delta-opioid receptor activation and microrna expression of the rat cortex in hypoxia. PLoS One 2012;7:e51524.

27 He X, Yang Y, Zhi F, Moore ML, Kang X, Chao D, Wang R, Balboni G, Salvadori S, Kim DH, Xia Y: $\Delta$-opioid receptor activation modified microrna expression in the rat kidney under prolonged hypoxia. PLoS One 2013;8:e61080.

28 Feng Y, He X, Yang Y, Chen J, Yin K, Xia Y: Effect of delta-opioid receptor over-expression on cortical expression of gabaa receptor alpha1-subunit in hypoxia. Chin J Physiol 2011;54:118-123.

29 Chao D, Bazzy-Asaad A, Balboni G, Xia Y: Delta-, but not mu-, opioid receptor stabilizes k(+) homeostasis by reducing $\mathrm{ca}(2+)$ influx in the cortex during acute hypoxia. J Cell Physiol 2007;212:60-67.

30 Liu B, Che W, Xue J, Zheng C, Tang K, Zhang J, Wen J, Xu Y: Sirt4 prevents hypoxia-induced apoptosis in h9c2 cardiomyoblast cells. Cell Physiol Biochem 2013;32:655-662.

31 Condorelli G, Latronico MV, Cavarretta E: Micrornas in cardiovascular diseases: Current knowledge and the road ahead. J Am Coll Cardiol 2014;63:2177-2187.

32 Xu Y, Zhu W, Sun Y, Wang Z, Yuan W, Du Z: Functional network analysis reveals versatile micrornas in human heart. Cell Physiol Biochem 2015;36:1628-1643.

33 Wang H, Bei Y, Shi J, Xiao J, Kong X: Non-coding rnas in cardiac aging. Cell Physiol Biochem 2015;36:16791687.

34 Wang J, Liew OW, Richards AM, Chen YT: Overview of micrornas in cardiac hypertrophy, fibrosis, and apoptosis. Int J Mol Sci DOI: 10.3390/ijms17050749.

35 Ha M, Kim VN: Regulation of microrna biogenesis. Nat Rev Mol Cell Biol 2014;15:509-524.

36 Li B, Li R, Zhang C, Bian HJ, Wang F, Xiao J, Liu SW, Yi W, Zhang MX, Wang SX, Zhang Y, Su GH, Ji XP: Microrna-7a/b protects against cardiac myocyte injury in ischemia/reperfusion by targeting poly(adpribose) polymerase. PLoS One 2014;9:e90096.

37 Meng S, Cao J, Wang L, Zhou Q, Li Y, Shen C, Zhang X, Wang C: Microrna 107 partly inhibits endothelial progenitor cells differentiation via hif-1beta. PLoS One 2012;7:e40323.

38 Voellenkle C, van Rooij J, Cappuzzello C, Greco S, Arcelli D, Di Vito L, Melillo G, Rigolini R, Costa E, Crea F, Capogrossi MC, Napolitano M, Martelli F: Microrna signatures in peripheral blood mononuclear cells of chronic heart failure patients. Physiol Genomics 2010;42:420-426.

39 Zen K, Zhang CY: Circulating micrornas: A novel class of biomarkers to diagnose and monitor human cancers. Med Res Rev 2012;32:326-348.

40 Baseler WA, Thapa D, Jagannathan R, Dabkowski ER, Croston TL, Hollander JM: Mir-141 as a regulator of the mitochondrial phosphate carrier (slc25a3) in the type 1 diabetic heart. Am J Physiol Cell Physiol 2012;303:C1244-1251.

41 Chan YC, Khanna S, Roy S, Sen CK: Mir-200b targets ets-1 and is down-regulated by hypoxia to induce angiogenic response of endothelial cells. J Biol Chem 2011;286:2047-2056. 


\section{Cellular Physiology Cell Physiol Biochem 2016;39:1118-1128 \begin{tabular}{l|l|l} 
DOI: 10.1159/000447815 29,2016 & $\begin{array}{l}\text { O 2016 The Author(s). Published by S. Karger AG, Basel } \\
\text { www.karger.com/cpb }\end{array}$
\end{tabular} \\ Zhi et al.: $\delta$-Opioid Receptor and MicroRNAs in Hypoxic Heart}

42 Barsanti C, Trivella MG, D'Aurizio R, El Baroudi M, Baumgart M, Groth M, Caruso R, Verde A, Botta L, Cozzi L, Pitto L: Differential regulation of micrornas in end-stage failing hearts is associated with left ventricular assist device unloading. Biomed Res Int 2015;2015:592512.

43 He F, Lv P, Zhao X, Wang X, Ma X, Meng W, Meng X, Dong S: Predictive value of circulating mir-328 and mir134 for acute myocardial infarction. Mol Cell Biochem 2014;394:137-144.

44 Wang KJ, Zhao X, Liu YZ, Zeng QT, Mao XB, Li SN, Zhang M, Jiang C, Zhou Y, Qian C, Feng KG, Guan HQ, Tang TT, Cheng X, Chen ZJ: Circulating mir-19b-3p, mir-134-5p and mir-186-5p are promising novel biomarkers for early diagnosis of acute myocardial infarction. Cell Physiol Biochem 2016;38:1015-1029.

45 Xiao J, Jing ZC, Ellinor PT, Liang D, Zhang H, Liu Y, Chen X, Pan L, Lyon R, Peng LY, Liang X, Sun Y, Popescu LM, Condorelli G, Chen YH: Microrna-134 as a potential plasma biomarker for the diagnosis of acute pulmonary embolism. J Transl Med 2011;9:159.

46 Ge Y, Pan S, Guan D, Yin H, Fan Y, Liu J, Zhang S, Zhang H, Feng L, Wang Y, Xu R, Yin JQ: Microrna-350 induces pathological heart hypertrophy by repressing both $\mathrm{p} 38$ and jnk pathways. Biochim Biophys Acta 2013;1832:1-10.

47 Chao D, Xia Y: Ionic storm in hypoxic/ischemic stress: Can opioid receptors subside it? Prog Neurobiol 2010;90:439-470.

48 Ma MC, Qian H, Ghassemi F, Zhao P, Xia Y: Oxygen-sensitive \{delta\}-opioid receptor-regulated survival and death signals: Novel insights into neuronal preconditioning and protection. J Biol Chem 2005;280:1620816218.

49 He X, Sandhu HK, Yang Y, Hua F, Belser N, Kim DH, Xia Y: Neuroprotection against hypoxia/ischemia: Deltaopioid receptor-mediated cellular/molecular events. Cell Mol Life Sci 2013;70:2291-2303.

50 Estrada JA, Williams AG Jr, Sun J, Gonzalez L, Downey HF, Caffrey JL, Mallet RT: Delta-opioid receptor (DOR) signaling and reactive oxygen species (ros) mediate intermittent hypoxia induced protection of canine myocardium. Basic Research in Cardiology DOI: 10.1007/s00395-016-0538-5.

51 Witman N, Heigwer J, Thaler B, Lui WO, Morrison JI: Mir-128 regulates non-myocyte hyperplasia, deposition of extracellular matrix and islet1 expression during newt cardiac regeneration. Dev Biol 2013;383:253-263. 